\title{
Nordiques
}

35 | 2018

Tove Jansson : Par delà les genres. Pratiques

linguistiques dans le Norden du XXIe siècle : Quels

enjeux sociaux?

\section{Entretien avec Christian Sébire, fondateur du parc historique Ornavik}

\section{Eric Eydoux}

\section{OpenEdition \\ Journals}

\section{Édition électronique}

URL : https://journals.openedition.org/nordiques/1068

ISSN : 2777-8479

Éditeur :

Association Norden, Bibliothèque de Caen la mer

\section{Édition imprimée}

Date de publication : 1 mai 2018

Pagination : 157-162

ISBN : 979-1-0959140-1-3

ISSN : $1761-7677$

Référence électronique

Eric Eydoux, «Entretien avec Christian Sébire, fondateur du parc historique Ornavik», Nordiques [En ligne], 35 | 2018, mis en ligne le 02 février 2021, consulté le 06 octobre 2021. URL : http://

journals.openedition.org/nordiques/1068 


\section{Entretien avec Christian Sébire, fondateur du parc historique Ornavik}

Les Normands aiment se référer à leur passé viking. En portent notamment témoignage les drakkars et autres casques cornus auxquels recourent si volontiers commerces et associations diverses. S'y ajoutent, durant la belle saison, ces éphémères campements vikings qui s'efforcent de faire revivre cette époque, le plus souvent pour en souligner la violence.

Parmi les initiatives des "reconstructeurs", il en est cependant une qui, se distinguant des autres, retient plus spécialement l'attention. S'inscrivant dans la durée, le parc historique Ornavik, aux portes de Caen, s'attache à restituer les conditions d'existence en Normandie de l'arrivée des Vikings à Guillaume le Conquérant. En utilisant les techniques et matériaux de l'époque, le chantier, en cours de réalisation, fait appel à de nombreux bénévoles. Il bénéficie cependant de la vétilleuse caution de scientifiques reconnus, dont ceux de l'université de Caen. En outre, il s'inscrit dans un réseau de quinze institutions européennes similaires. Pour en savoir plus, nous avons interrogé Christian Sébire, le président fondateur de l'association.

Éric Eydoux : Dans le paysage culturel et touristique de la Normandie, le parc historique Ornavik, un nom aux résonances scandinaves choisi à dessein, occupe maintenant une place notable. Christian Sébire, vous en êtes le fondateur et le président. Comment avez-vous été amené à concevoir et mettre en œuvre ce projet?

Christian Sébire : En 2011, à l'occasion du onze centième anniversaire de la Normandie, il a paru souhaitable de fonder un parc historique viking qui raconterait la fabuleuse histoire de sa naissance, depuis Rollon le Viking jusqu’à Guillaume le Conquérant, c’est-à-dire des années 911 à 1066.

E. E. : Vous souhaitiez donc remonter aux origines?

C. S. : En fait, à l'époque du cinquantième anniversaire du débarquement, alors que je siégeais à l'office de tourisme, il nous était apparu que, sur le plan touristique, nous étions trop tributaires des vétérans de la dernière guerre et que, ceux-ci se faisant de moins en moins nombreux, il devenait nécessaire de trouver un autre axe de développement. Sous ce rapport, Guillaume le Conquérant était un thème de choix. Néanmoins, cette grande figure historique était surtout connue des 
Anglais et des Normands et il convenait de viser un public plus large qui permettrait d'autofinancer le parc. Dans cet esprit, le thème des Vikings satisfaisait tout à la fois aux exigences de l'histoire et du marketing.

E. E. : Mais n'êtes-vous pas vous-même un professionnel du tourisme?

C. $S$. : Non, je suis photographe de formation et me suis ensuite tourné vers le monde de l'industrie. Pour autant, même si je ne suis pas un professionnel, je me suis rapidement passionné à la fois pour le tourisme et l'histoire.

E. E. : Cette aventure, vous l'avez commencée seul, mais, dans sa phase initiale, y a-t-il des personnes qui vous ont apporté leur concours?

C. S. : Je me dois d'abord de rendre hommage à Christian Heitz, bien connu à Caen pour sa boulangerie. C'est grâce à lui qu'Ornavik a pu voir le jour. J'avais déjà un dossier dans mes tiroirs lorsqu'en 2008, Christian Heitz a été élu à la ville d'Hérouville. Et quand le maire, Rodolphe Thomas, lui a fait découvrir la ville et le domaine de Beauregard, appelé à être valorisé pour devenir une zone de loisirs, Christian s'est rappelé le dossier viking et a fait le rapprochement. Voilà comment l'histoire a commencé.

E. E. : Ce domaine de Beauregard est extrêmement vaste. Quelles sont ses dimensions?

C. S. : Le domaine couvre une superficie de 17 hectares et la ville a accepté de nous en louer dix à seule fin de l'utiliser pour raconter l'histoire de la Normandie. Ce site était idéal puisqu'il offrait les trois types de paysage qui nous étaient nécessaires ; une plaine, une forêt et une partie basse bordée de plans d'eau. Cela correspondait aux trois espaces que nous souhaitions aménager : un espace carolingien donnant un aspect de notre région avant que les Vikings ne l'envahissent. C'est l'espace dit du plateau, par lequel nous entrons dans le parc. Ensuite, nous traversons la forêt pour gagner la zone humide où a été aménagé le site viking. Après quoi, on rejoint le troisième espace où commence à s'élever une motte castrale. Les visiteurs connaissent bien les châteaux de Falaise et de Caen. Or ces magnifiques bâtiments en pierre étaient l'apanage des ducs et, une fois leur aval obtenu, les seigneurs ne pouvaient que faire construire ces mottes castrales beaucoup plus modestes dont nous avons jugé bon de reconstituer un exemplaire.

$E$. E. : Lorsque vous avez lancé ce projet, vous n’avez bénéficié d'aucun soutien officiel ?

C. S. : Non, car je pense que si nous l'avions demandé, nous ne l'aurions pas obtenu. Mais surtout, l'idée sous-jacente était que ce projet devait être porté par les Normands. Pour qu'il soit une réussite, il fallait que ceux-ci s'en emparent. Cela signifie en d'autres termes que, refusant les subventions, nous comptions sur 
une participation directe de la population. Il pouvait s'agir de personnes morales, d'entreprises prêtes à financer le projet par le biais du mécénat ou encore de personnes physiques disposées à participer bénévolement à ce grand œuvre. Et c'est bien ce qui s'est passé. Aujourd'hui, Ornavik a plus de soixante entreprises partenaires auxquelles trois ou quatre viennent s'ajouter chaque mois. Le processus va s'accélérant et de même en va-t-il des bénévoles. On en compte actuellement entre 180 et 200 avec une moyenne de $10 \%$ supplémentaires chaque année.

$E$. E. : D'évidence, le succès appelle le succès et l'on ne peut manquer d'être impressionné lorsqu'on constate ce que vous avez pu obtenir de ces entreprises comme celles des travaux publics qui ont bouleversé l'aspect initial du domaine de Beauregard. Tout comme la main-d'œuvre occupée aux tâches les plus diverses. Mais cette même main-d'œuvre est-elle uniquement bénévole ou bénéficiez-vous aussi d'emplois aidés?

C. S. : Ce sont des bénévoles qui construisent Ornavik. Pour autant, c'est avec grand plaisir que nous recevons des structures d'insertion qui trouvent là une occasion extraordinaire de motiver ces jeunes très souvent déstructurés et en grande difficulté. C'est d'ailleurs beaucoup plus facile pour eux de travailler avec des bénévoles plus à l'écoute et plus compréhensifs que le personnel d'entreprises commerciales. Grâce à un projet comme Ornavik, ces jeunes peuvent effectuer un travail extrêmement valorisant. Considérez, par exemple, les portails en chêne qui marquent l'entrée du parc historique. Ils ont été réalisés par des chantiers d'insertion. Imaginez le plaisir ressenti par ces jeunes qui ont construit ces magnifiques portails et peuvent recevoir famille et amis en utilisant une carte valable à vie pour se rendre à Ornavik. Voilà qui fonctionne très bien.

E. E. : Vous reconstituez des bâtiments et objets de ces différentes époques en faisant toujours preuve d'une grande rigueur scientifique. Avez-vous recours à des spécialistes?

C. S. : Oui, nous ne voulons pas être un parc d'attractions. On nous dit fréquemment : "Vous allez être un Puy du Fou normand. " Mais telle n'est pas notre ambition. Ce que nous voulons, c'est mettre le visiteur en immersion au Moyen Âge. Nous souhaitons impérativement que chaque maison, chaque plante, chaque vêtement, chaque outil soient une réplique aussi exacte que possible de ce qui a existé. Pour s'en assurer, il fallait s'entourer de personnes compétentes. Et, à Caen, nous avons la chance d'avoir une université qui, avec le CRAHAM (Centre de recherches archéologiques et historiques anciennes et médiévales), rassemble les meilleurs spécialistes. S’y ajoute un service départemental d'archéologie qui nous a apporté son précieux concours ainsi que plusieurs autres. Au total, ce ne sont pas moins de onze archéologues qui siègent dans notre conseil scientifique. 
E. E. : Outre cette rigueur scientifique, vous avez eu le grand mérite de faire revivre un certain nombre de métiers que l'on aurait pu croire obsolètes ou tombés en désuétude, telle la construction de toits de chaume. Pouvez-vous nous en dire plus sur ces métiers?

C. S. : Il s'agit de tous les métiers liés à la culture et à la construction. Quand on édifie une maison, on construit les murs soit en terre soit en torchis. Il a donc fallu réapprendre à faire des clayonnages, c'est-à-dire croiser des branches de noisetier pour créer une structure puis, de chaque côté, élever des murs de terre mêlée de foin mouillé. Préalablement, il avait fallu réaliser la charpente, non avec du bois équarri, mais avec des troncs, ce qui est beaucoup plus difficile. Pour terminer, on pose des toits de bardeaux, souvent avec des tuiles en châtaigner, à moins que l'on opte pour des toits de chaume, principalement avec du roseau. Car au Moyen Âge, on n'allait pas chercher des matériaux à des dizaines, voire des centaines de kilomètres. On recourait aux matériaux disponibles sur place et nous faisons de même à Ornavik. Tous ces métiers, il a donc fallu les apprendre ou les réapprendre. Et l'une des principales ambitions d'Ornavik au cours des prochaines années est de devenir un véritable conservatoire des anciens savoirfaire. Nous nous dirigeons à grands pas vers la numérisation, la robotisation, l'automatisation et risquons corrélativement de voir se perdre d'ancestraux savoirfaire. La volonté d'Ornavik de remettre ces techniques à l'honneur arrive à point nommé, à un moment où des aînés sont encore là avec leur savoir-faire et parfois leurs outils. Mieux vaut ne pas tarder.

E. E. : En complément à ces projets, en aboutissement faudrait-il peut-être dire, vous prévoyez la construction d'un Centre d'interprétation viking. De quoi s'agit-il ?

C. S. : Le pourquoi de ce centre ? Nous avons un parc historique qui, avec plus de 30000 visiteurs depuis sa création, se développe de manière aussi satisfaisante que possible. Néanmoins, nous n'avons pas de bâtiment d'accueil, pourtant indispensable si nous voulons développer la partie touristique, le Centre d'interprétation viking proprement dit qui a toute sa raison d'être. En effet, il se trouve qu'en Scandinavie, comme dans d'autres pays d'Europe, il existe de nombreux musées consacrés aux Vikings. Généralement, ils sont très bien faits, mais restent des musées. La différence est que, dans un musée, on trouve des artefacts, des bateaux, du mobilier. À titre d'exemple, le musée de Roskilde, au Danemark, raconte l'histoire des Vikings à partir de ses trois bateaux retrouvés dans le fjord. Mais aucun de ces musées ne raconte l'histoire des Vikings du début à la fin des expéditions, c'est-à-dire du VIII ${ }^{e}$ au X $\mathrm{X}^{\mathrm{e}}$ siècle. Et si, en Normandie, nous avons trouvé pertinent de créer ce centre d'interprétation, c'est que, nulle part ailleurs, le thème des Vikings n'y rencontre un écho aussi favorable. Actuellement, nous 
avons sollicité un cabinet spécialisé dans le tourisme et la culture pour faire des études préparatoires et nous travaillons avec eux sur le dimensionnement du bâtiment. Les résultats devraient être connus au mois de septembre. Le centre aura-t-il une dimension internationale, nationale ou régionale ? Ce sera d'abord un choix politique qui ne nous appartient plus.

E. E. : Un des aspects les plus intéressants de votre projet est qu'il s'inscrit dans une chaîne de centres comparables. Avec combien de pays avez-vous ainsi noué des partenariats?

C. S. : Nous faisons partie d'une association qui s'appelle Destination Viking et qui regroupe quinze pays d'Europe ainsi que la Russie et le Canada. Financée par l'Europe, elle rend compte de la route des Vikings, et nous sommes ses représentants en France. Grâce à cette structure, nous pouvons échanger avec les spécialistes de l'époque viking des différents pays et ainsi nourrir nos projets, car, en France, nous n'avons aucune trace de constructions vikings et les témoignages archéologiques ne sont guère probants. Hormis quelques armes, deux tombes dont celle, naviforme, de l'île de Groix, il n'existe pratiquement rien. Il était donc indispensable de prendre l'attache des pays européens.

E. E. : Maintenant que vous avez ainsi avancé, les pouvoirs publics sont-ils disposés à mieux vous aider ?

C. $S$. : Au cours des dix-huit derniers mois, nous avons invité l'ensemble des collectivités à nous rejoindre et réussi à les convaincre de la faisabilité du projet. Depuis, celles-ci nous aident en investissement, la Région en tête, suivie de Caen la mer, du département du Calvados, de la ville d'Hérouville-Saint-Clair et aussi celle de Mondeville où ont lieu les fouilles du village carolingien qui s'appelait Saint-Martin de Trainecourt. Oui, nous sommes suivis, bien accompagnés par les collectivités.

E. E. : Vous avez parlé du nombre de visiteurs que vous avez accueillis. Trente mille cette année?

C. S. : Non, pas cette année, mais depuis l'ouverture. Et il ne faut pas oublier que nous ne sommes ouverts que quelques dimanches par an puisque ce sont des bénévoles qui assurent les ouvertures et que, déjà, ils travaillent toute l'année à l'aménagement du parc Ornavik. Il n'était pas envisageable qu'ils viennent en plus travailler les week-ends pendant six mois afin d'ouvrir et de faire vivre le parc. Une des grandes originalités d'Ornavik, de son succès aussi, tient à ce que ce soit un parc de plein air vivant : vous voyez le forgeron forger, l'agriculteur travailler aux champs et le bâtisseur construire sa maison. 
E. E. : Arriverez-vous à ouvrir pendant toute la saison d'été ?

C. S. : Jusqu'en 2021, nous n'aurons pas la possibilité d'ouvrir comme un site touristique digne de ce nom, c'est-à-dire, pour les raisons évoquées plus haut, dès début avril et jusqu'à fin septembre. Mais, à partir de 2021, c'est le Centre d'interprétation viking qui nous permettra d'avoir des salariés en plus des bénévoles et de pouvoir fonctionner comme un vrai site touristique.

E. E. : De l'avis général, avec énergie et intelligence, vous avez mis en œuvre un projet d'exception voué à la réussite. Mais il est encore une récente initiative qui, de nature à élargir sensiblement votre audience, notamment auprès des pays partenaires, mérite d'être mentionnée.

C. S. : Le 28 avril prochain, le marathonien Lionel Rivoire va se rendre à Aalesund, en Norvège, là où Rollon est censé être né. Il va ensuite parcourir le chemin que ce dernier a pu emprunter : d'Aalesund à Bergen, de Bergen à Oslo, d'Oslo à Trelleborg (Suède), de Trelleborg à Roskilde (Danemark), de Roskilde à Lejre, de Lejre à Jelling, de Jelling à Ribe et enfin Haithabu (anciennement Hedeby) en Allemagne. Cette aventure pourra être suivie par tous les Normands sur un site dédié (<www.rollon2018.fr>), où Lionel Rivoire relatera tous les soirs son périple. On notera d'ailleurs que, le 27 avril, la veille de son départ, la région de Normandie lancera sa propre monnaie baptisée le « Rollon ». 